\title{
Analysis of EEG Rhythms in Epilepsy Patients Using MPSO Method
}

\author{
M.J.S Joshi ${ }^{1}$, R.S.K Vaibhav ${ }^{2}$, R.V.S Satyanarayana ${ }^{3}$ \\ PG Student [Comm. Systems], Dept. of ECE, S.V.U College of Engg, S.V. University, Tirupati ${ }^{1}$ \\ Dept. of ECE, S.V. College of Engg, Tirupati ${ }^{2}$ \\ Professor, Dept. of ECE, S.V.U College of Engg, S.V. University, Tirupati ${ }^{3}$
}

\begin{abstract}
One of the most important things in diagnosis of epilepsy is to find the exact location of the epileptogenic point. EEG is a tool commonly used at epilepsy diagnosis centers for diagnosis purposes. . In this paper, the modified particle swarm optimization (MPSO) method used to solve the EEG source localization problem. The attempt has been done here to estimate the brain activity on the basis of spectrum analysis. EEG classification can be very useful in predicting the action or the intention of action performed on the basis of EEG which leads to more development in brain computer interface. The brain waves $\alpha, \beta, \Upsilon, \delta, \Theta$ were extracted using frequency filtering and estimating the level of disease is done by the clinical experts based on the amplitudes of the brain waves. The EEG data has been referred from a website MIT-BIH and the mathematical tool for EEG analysis called EEGLAB has been used to perform work in this paper.
\end{abstract}

Index Terms: Electroencephalogram (EEG), particle swarm optimization (PSO).

\section{INTRODUCTION}

Epilepsy is one of the most common brain diseases in the used in the present study was recorded from evoked world. Epilepsy is brief re-current disorder of cerebral potentials stimulation on a healthy subject. In this paper, function that is usually associated with disturbances of EEG signal of visual attention task have been analyzed. consciousness and accompanied by a sudden, excessive For the EEG data set of 32 channels has been used .Firstly, electrical discharge of cerebral neurons. EEG is of high the signal has been filtered to remove artifacts from the voltage relative to the background and results from an un- signal.

physiological synchronous discharge of aggregation of neurons. Clinically, epilepsy is defined as a condition characterized by the recurrent two or more unprovoked seizures. The source localization of epileptic activity is a tool to delineate cortical areas/volumes with abnormal neuronal condition in which the things are happen of cells and networks. However, correct and anatomically precise localization of the epileptic focus is compulsory to decide if resection of brain tissue is possible. Present day computer systems can be used for the time domain visualization of multi-channel EEG data.

It is well known fact that the Electroencephalogram (EEG) signals are the measure of the vigilance state of brain which

Changes according to task performed by a person. These changes are classified into few different frequency bands named as delta, theta, alpha, beta [2].The accurate classification of electrical activity for a particular state of human brain helps in neurological diagnosis. The procedure of the EEG source localization deals with two problems: 1) a forward method to find the scalp possibilities for the given current source(s) inside the brain, 2) an inverse problem to assess the place of origin that appropriate with the given latent qualities or possibilities distribution at the scalp electrodes.[1] In this paper, the MPSO method is applied to EEG data. Resolving the EEG source localization problem in the daily routine laboratory application. The EEG data that is

\section{EEG Patterns in Brain}

1. Delta $(\delta)$

Frequency: 0.5 to $4 \mathrm{~Hz}$

Occurrence: This occur only once in every 2 or 3 seconds. These occur in deep sleep, in premature babies and in very serious organic brain diseases. These can occur strictly in the cortex independently by the activities in the lower regions of brain.

\section{Theta $(\theta)$}

Frequency: 4 to $8 \mathrm{~Hz}$

Occurrence: these are recorded from the parietal and temporal regions of the scalp of children. These also occur during emotional stress in some adults particularly during disappointment and frustration.

3. Alpha ( $\alpha)$ :

Frequency: 8 to $13 \mathrm{~Hz}$

Occurrence: they found in normal persons when they are awake in quite, resting state. They occur normally occipital region.

\section{Beta ( $\beta$ )}

Frequency: 13 to $30 \mathrm{~Hz}$ (At intense mental activity, the frequency increases up to $50 \mathrm{~Hz}$ )

Occurrence: These are recorded from the parietal and frontal regions of the scalp. These are divided into two types as beta I which is inhibited by the cerebral activity and beta II which is excited by the mental activity, like tension. 


\section{Different Stages of Sleep Pattern}

Analysis of EEG done to classify different stages of sleep depends upon the percentage power in each band.

Different stages sleeps are:

- Alert, wide awake person displays the unsynchronized high frequency EEG

- Drowsy person, one whose eyes are closed, it produces large amount regularly activity in the range 8 to $13 \mathrm{~Hz}$

- The person begins to full sleep then the amplitude and frequency of the wave form decrease.

- Light sleep, it is large amplitude and low frequency of the wave form emerges.

- Deep sleep, then its results is even slower and higher amplitude waves [3].

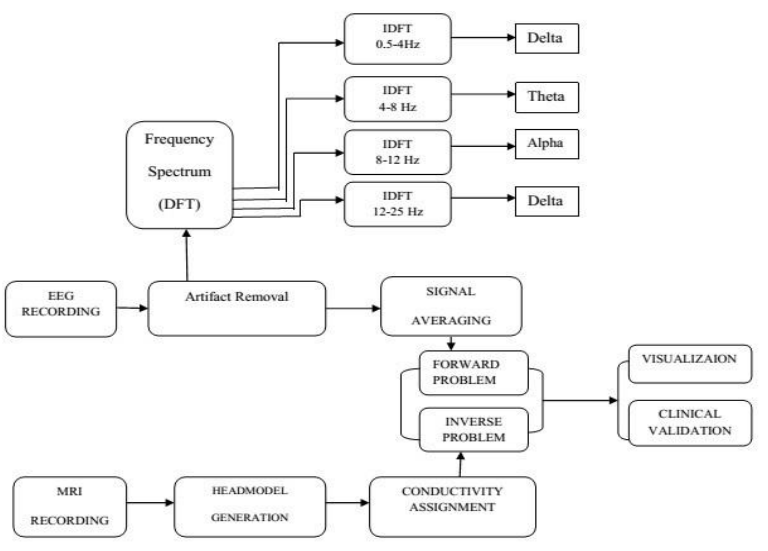

Fig1.Block Diagram of EEG source localization procedure

\section{MRI AND EEG DATA}

Structural MR Images were acquired using a PHILIPS ACHIEVA 3T scanner (GE medical system database) equipped with a 32 channel head coil and EEG data is taken from MITBIH data base. T1-weighted images were acquired for a healthy subject, (192 sagittal slices, matrix size $=256 \times 256$, voxel size $=0.9375 \mathrm{~mm}^{3}$, flip angle $8^{0}$, $\mathrm{TR} / \mathrm{TE}=2.300 / 2.98 \mathrm{~ms}$ ). The segmentation of the five tissues, i.e., cerebrospinal fluid (CSF), white matter, grey matter, skull and skin, was done by FSL. The segmented tissues were checked and corrected manually by a clinical expert. Here the high resolution model is necessary to accurately model the CSF compartment and the thin area of the skull. We generated the $1 \mathrm{~mm}$ hexahedra FE head model with approximately 2.8 million cells from segmented MRI, see Fig. 2. The following conductivities were then assigned to the FE compartments based on their segmentation labels and the isotropic reference model: skin $=0.43 \mathrm{~S} / \mathrm{m}$, skull $=0.0042 \mathrm{~S} / \mathrm{m}$ (skull to skin conductivity ratio of approximately $1: 100), \mathrm{CSF}=1.538$ $\mathrm{S} / \mathrm{m}$, grey matter $=0.33 \mathrm{~S} / \mathrm{m}$ and white matter $=0.142$ $\mathrm{S} / \mathrm{m}$. The 32 EEG electrodes were placed on the subject's head based on the 10/10 EEG electrode system.

\section{III.COMPUTATIONS}

The following steps are involved in this work shown in the Figure1.Starting from loading the EEG signals of recorded
EEG signals has been done in the range of frequencies $0.1 \mathrm{~Hz}$ to $60 \mathrm{~Hz}$ by applying FIR filter of second order. At the same time notch filter is also used to remove the power line interface in the channels spectrum around $50 \mathrm{~Hz}$. The artifacts are the disturbances in all or few channels due to eye blink or sometime by the low conductivity of electrodes. These artifacts are trimmed off manually from the signals all channels at once. After applying the DFT or FFT to an EEG signal, the magnitude values are stored for each band. The lower cut of frequency and upper cut of frequency is defined depends upon the frequency range of each band only the signals between the upper band and lower band of EEG remain as it is and others magnitudes are zero padded., for plotting graphical results for each band like Delta, Alpha, Beta and Theta for expressions of power in each bands.

The Discrete Fourier Transform is given by

$$
X(k)=\sum_{n=0}^{N-1} x(n) e^{-j 2 \pi k n / N} n=0,1,2 \ldots \mathrm{N}-1 \ldots
$$

The inverse Discrete Fourier is given by

$$
x(n)=\frac{1}{N} \sum_{k=0}^{N-1} \mathrm{X}(\mathrm{k}) e^{j 2 \pi n t / N} k=0,1,2 \ldots N-1 \ldots
$$

It is important to note that zero padding does not provide any additional information about the spectrum $x(w)$ of the sequence $x(n)$.How ever padding the sequence $x(n)$ with $\mathrm{N}-\mathrm{L}$ Zero and computing an $\mathrm{N}$ Point DFT results in a better display of the Fourier frequency $\mathrm{x}(\mathrm{w})$.

$$
x(k)=\sum_{n=0}^{N-1} x(n) W_{N}^{k n} \ldots
$$

Where $W_{N}=e^{-j 2 \pi / N}$

$\mathrm{N}=\mathrm{N}$ Point DFT

$\mathrm{L}=$ Total number of samples

To reduce the number of computations like addition and multiplications over DFT, the most powerful transform is FFT is used for the computation of FFT the decimation into smaller value. We can generalize the relations for the first two decompositions of Decimations in time FFT algorithms. It can be written as,

$$
\begin{gathered}
\mathrm{X}_{m-r, p}(k)=X_{m-r-1,2 p-1}(k)+W_{N}^{2 r k} X_{m-r-1,2 p}(k) \ldots(4) \\
X_{m-r, p}\left(k+N / 2^{r-1}\right)=X_{m-r-1,2 p-1}(k)+W_{N}^{2 r k} X_{m-r-1,2 p}(k) \ldots(5)
\end{gathered}
$$

The power spectrum is computed which is given by formula

$$
P x=1 / N \sum_{n=0}^{N-1}|X(\mathrm{n})|^{2} \ldots(6)
$$

A. Particle Swarm Optimization (PSO) Algorithm: The PSO is an existing method used to localize the EEG signal on the brain model. The algorithm for the PSO is as follows. [1]

Step 1: Initialize the population - locations and velocities Step 2: Evaluate the fitness of the individual particle Step 3: Keep track of the individual's highest fitness 
Step 4: Modify velocities based on best position

Step 5: Update the particles position

Step 6: Terminate if the condition is met

Step 7: Go to Step 2

\section{Modified Particle Swarm Optimization (MPSO):}

The Modified Particle Swarm Optimization is used to source localization of epilepsy foci path. In this method M particles are selected among the swarm population by the q-competition selection method and then mutated by the EP method. By evaluating the aptness value of all the atoms, the global best position is resolute. For each particles, the nearest best particle is determined by the geometer distance. The velocity and the position of the particles are updated according to the global best position, the nearest best position, and the personal best position.

These are applied to the PSO with inertia weight as follows:

$$
\begin{aligned}
V_{i}^{t+1}=\omega V_{i}^{t}+ & c_{1} \operatorname{Rand}()\left(P_{i}-x_{i}^{t}\right)+c_{2} \operatorname{Rand}()\left(P_{g}-x_{i}^{t}\right)+ \\
& c_{3} \operatorname{Rand}()\left(P_{e}-x_{i}^{t}\right) \quad \ldots(7)
\end{aligned}
$$

where $c_{3}$ denotes the constant of the nearest elite and $\mathrm{P}_{\mathrm{e}}$ the nearest elite position.

In the MPSO, we introduced the concept of expert to maintain the exploration ability and increase the exploitation ability. In the concept of expert, as the swarm moves close to lowest the $\mathrm{R}$ closest atoms to gbest are extracted and they are allowed to fly freely based on their storage and knowledge. Thus, the velocity update is divided into two parts as

$$
\begin{aligned}
V_{i}^{t+1}=\omega V_{i}^{t}+ & c_{1} \operatorname{Rand}()\left(P_{i}-x_{i}^{t}\right)+c_{2} \operatorname{Rand}()\left(P_{g}-x_{i}^{t}\right)+ \\
& c_{3} \operatorname{Rand}()\left(P_{e}-x_{i}^{t}\right) \ldots(8)
\end{aligned}
$$

where $\mathrm{i}=1,2, \ldots, \mathrm{N}-\mathrm{R}$ and

$$
\mathrm{V}_{\mathrm{r}}^{\mathrm{t}+1}=\omega \mathrm{V}_{\mathrm{r}}^{\mathrm{t}}+\mathrm{c}_{1} \operatorname{Rand}\left(\mathrm{P}\left(\mathrm{P}_{\mathrm{r}}-\mathrm{x}_{\mathrm{r}}^{\mathrm{t}}\right)\right.
$$

where $V_{r}, X_{r}$, and $P_{r}$ are the velocity, position and personal best of the th particle, respectively, for $\mathrm{r}=\mathrm{N}-\mathrm{R}+1, \ldots, \mathrm{N}$. The $\mathrm{R}$ nearest particles to gbest are reselected in each iteration to ensure that the particles which actuated away from the gbest mislay their authority and next repetitions update their velocity based on. It means that in some steps the mites which are closer to the global best can influence the performance and decision of the swarm more than others. The concept of experts allows the swarm to have more information around gbest before loads of particles come close to it and jammed with each other, thus it improves the exploitation ability. The concept of master mixed with EP helps to keep the balance of the investigation-exploitation trade-off as well as to avoid trapping in local minima. A natural choice for $\mathrm{R}$ is the number of neighborhoods for each particle in the swarm, which in our case is equal to 5 . The concept of authority allows the swarm to have more information around gbest before lots of particles approach it and get stuck to each other, thus it improves the exploitation ability.

\section{B. Validation}

Validation of the source localization is difficult, because there exist no "ground truth" to compare with and also we do not have access to other functional modalities, e.g.,
fMRI and MEG data from our subject for the same kind of stimulation. We have taken two approaches to validate our method: first we use the physiological knowledge on localization of motor and sensory functions based on clinical expertise and second we do an exhaustive search pattern, i.e., inversion was performed for each possible source location in the motor and sensory cortex area inside a region of interest (ROI), and the location producing the smallest residual norm was selected as the best possible source location. For the single point source the cost function (relative error) is defined as

$$
\text { Relative Error }=\frac{\left\|\mathrm{u}_{\text {meas }}-\mathrm{u}_{\mathrm{est}}\right\|}{\left\|\mathrm{u}_{\text {meas }}\right\|} \ldots
$$

where $u_{\text {meas }}$ is a vector of the measured potentials on the scalp, $\mathrm{u}_{\mathrm{est}}$ is a vector of the potential generated by the estimated dipole and $\|$.$\| denotes the Euclidean norm. For$ the exhaustive search we selected a large area that covered the entire left and right hemisphere motor and somatosensory cortex.
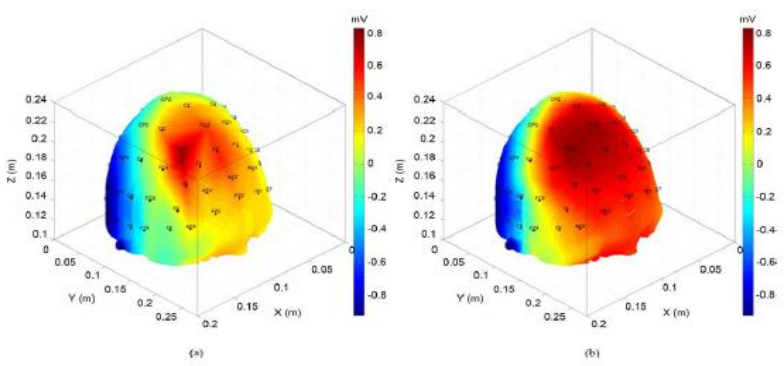

Fig2. Scalp surface potential topography for the median nerve stimulation (N20) (a) Measured EEG and (b) estimated EEG potential.

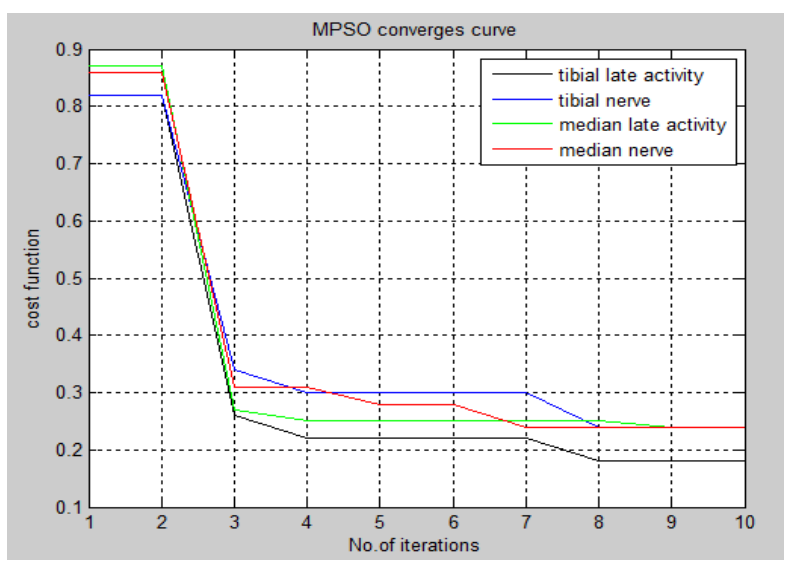

Fig3. MPSO convergence curve

\begin{tabular}{|c|c|c|c|c|}
\hline $\begin{array}{c}\text { No.of eval.for } \\
\text { MPSO }\end{array}$ & 300 & 300 & 310 & 320 \\
\hline $\begin{array}{c}\text { No.of eval. For } \\
\text { Exh.search }\end{array}$ & 110.00 & 110.00 & 110.00 & 110.00 \\
\hline Relative error & 0.2301 & 0.2307 & 0.1973 & 0.1834 \\
\hline
\end{tabular}

TABLE I. Number of Valuations and Relative Error for both the MPSO and Exhaustive Search Methods MPSO and Exhaustive Search Gave Identical Result But With A Much Lower Number of Evaluation For MPSO 


\section{RESULTS}

\section{A. Analytical results:}

The patient's data are stored in the disk under different conditions eyes open, eyes closed, epileptic patients, person awake, thinking, deep sleep. For each data file the Discrete Fourier Transform (DFT) is applied to calculate the magnitude of 8 channels. For better accuracy $\mathrm{N}=512$ is used as a $\mathrm{N}$ point DFT, for example a data file containing 5000 samples of 8 channels, the sample numbers 5000 to 5120 are zero padded and the data files for each channel contains the total samples 5120, for the file containing 5120 samples, the total windows are 10. After applying DFT to each patients file, the data is processed through different filters of each band to calculate the rhythm in each band. The table.II shows the rhythms for patients file. From the rhythms the depth of sleep is classified like Alert, drowsy sleep, light sleep and deep sleep.

\begin{tabular}{|c|c|c|c|}
\hline Rhythm & Frequency & Amplitude & $\begin{array}{l}\text { Conduction } \\
\text { Velocity }\end{array}$ \\
\hline Beta & $12-25 \mathrm{~Hz}$ & $18 \mu \mathrm{V}$ & $30-70 \mathrm{~m} / \mathrm{s}$ \\
\hline Alpha & $8-12 \mathrm{~Hz}$ & $6.8 \mu \mathrm{V}$ & $70-120 \mathrm{~m} / \mathrm{s}$ \\
\hline Theta & $4-8 \mathrm{~Hz}$ & $9.42 \mu \mathrm{V}$ & $3-15 \mathrm{~m} / \mathrm{s}$ \\
\hline Delta & $0.5-4 \mathrm{~Hz}$ & $52.8 \mu \mathrm{V}$ & $12-30 \mathrm{~m} / \mathrm{s}$ \\
\hline
\end{tabular}

Table II: Brain wave parameters of patient's EEG data

\section{B. Graphical Results}

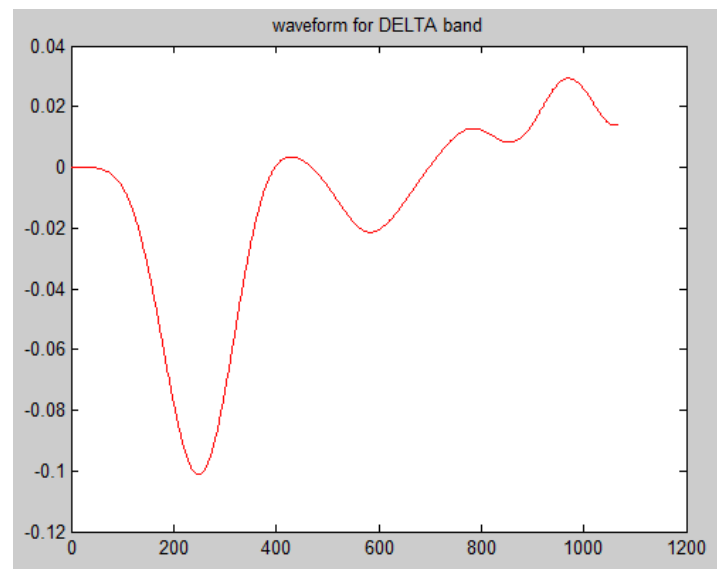

Fig4. Filtered Delta activity.

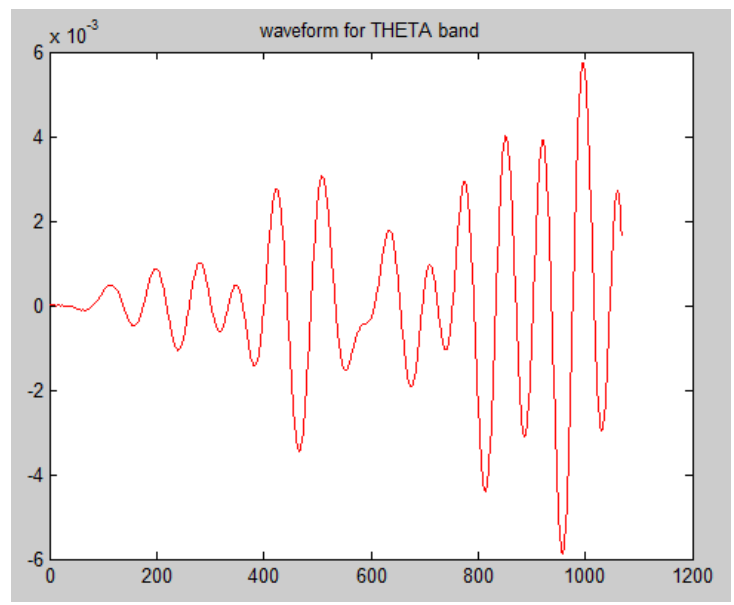

Fig5. Filtered Theta activity
For each of the EEG data files the DFT is applied. After application of DFT the data file is processed with different filters. The filters upper band and lower band depends upon the frequency range of each band.

Only the signal between these frequencies is passed and other signals are zero padded. After zero padding, Inverse Discrete Fourier Transform is applied to obtain the delta, alpha, Beta and Theta activity.

The graphical result are obtained window wise and channel wise or for total files. Fig.4, fig.5 and fig.6 show the output windows showing the Delta, Theta, Alpha. This makes the analysis easy and one can get the clear-cut idea about different frequency bands present in data.

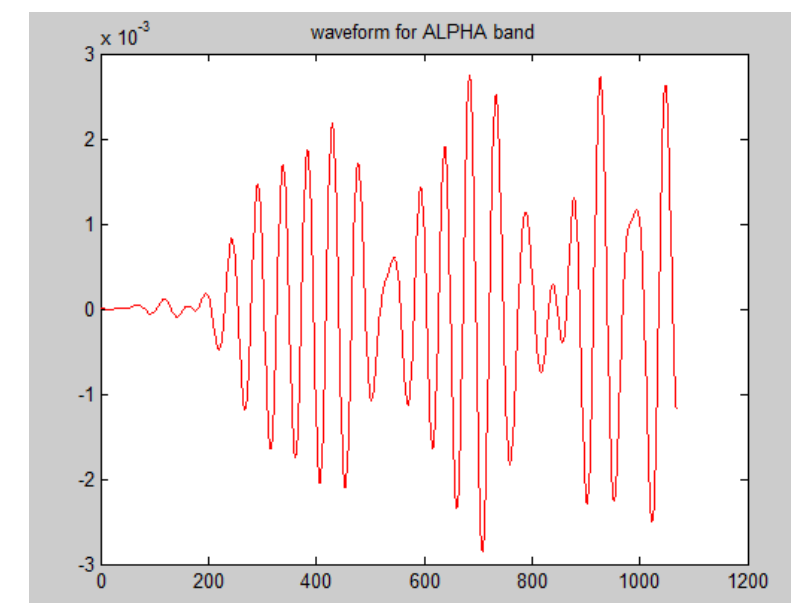

Fig7. Filtered Alpha activities

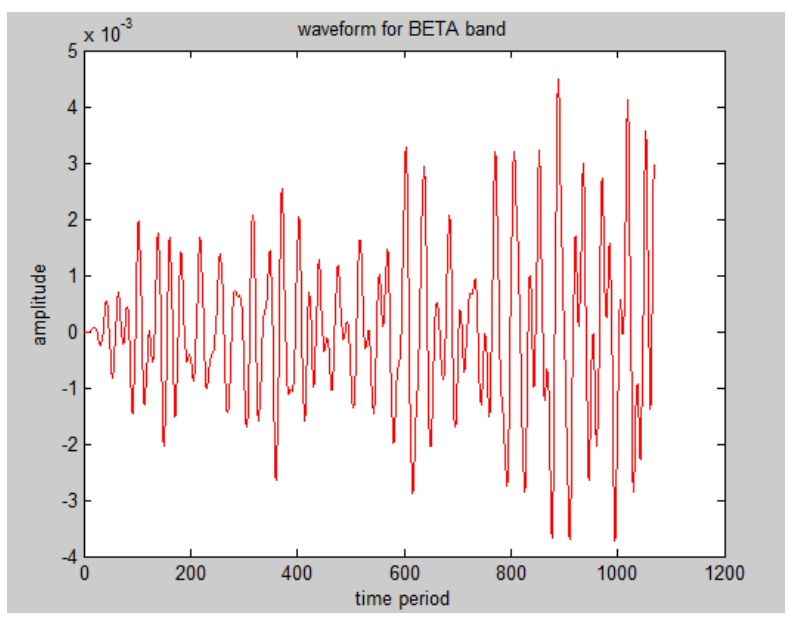

Fig8. Filtered Beta activities
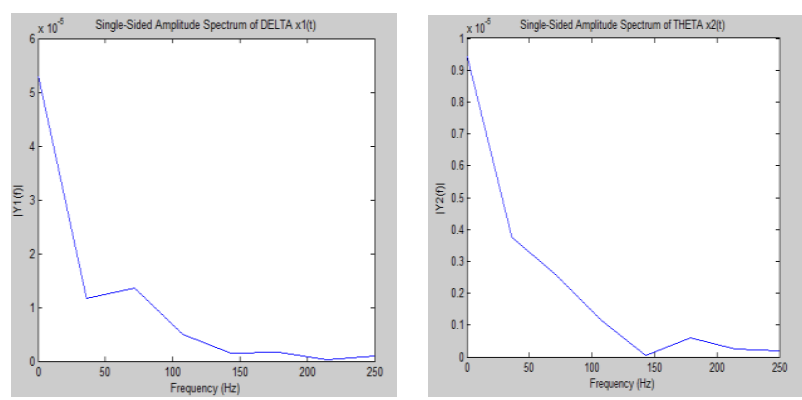
International Journal of Advanced Research in Computer and Communication Engineering Vol. 4, Issue 11, November 2015
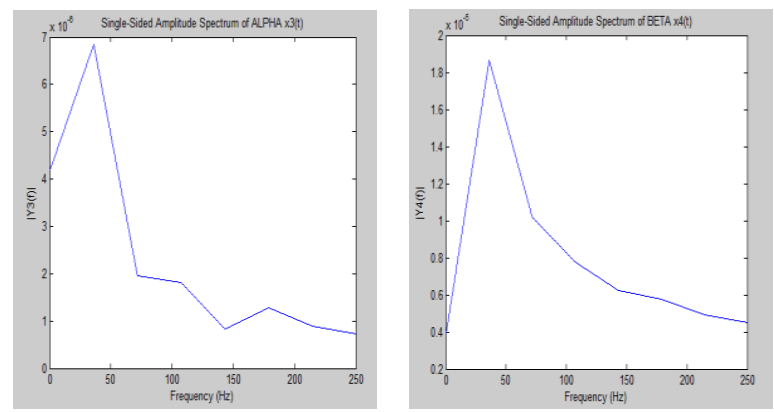

Fig8. Amplitude Spectrum of Dominant Brain Rhythms

\section{CONCLUSION}

In this paper EEG source localization is accomplished by using optimization technique called modified particles swarm optimization. In forward model reconstructed realistic high resolution finite element head volume conductor based on the T1 weight MR data set for construction of tissue model. An exhaustive search pattern is performed for each possible source localization. The localization results which is achieved by applying MPSO is similar to exhaustive search pattern, but with low complexity. The brain waves delta, theta, alpha, and beta are extracted by applying the frequency filtering .we estimate the dominant frequency in epilepsy origin point at source localize path. After extraction of brain waves and locating the origin point, person who are in the professions that needs to perform visual attention task more than normal person may be victim of defects in visual or cognitive tasks after a certain period of time.

\section{REFERENCES}

[1] Yazdan Shirvany, Student Member, IEEE, Qaiser Mahmood, Student Member, IEEE, Fredrik Edelvik, Stefan Jakobsson, Anders Hedström, and Mikael Persson, Member, IEEE, "Particle Swarm Optimization Applied to EEG Source Localization of Somatosensory Evoked Potentials", IEEE Transactions on Neural Systems and Rehabilitation Engineering, Vol. 22, No. 1, January 2014.

[2] Davidson's, "Principles and Practice of Medicine" $21^{\text {st }}$ Edition ,2010.

[3] L. Prakasam Reddy," Fundamentals of Medical Physiology" $4^{\text {th }}$ Edition, 2008.

[4] H. Laufs, "Functional imaging of seizures and epilepsy: Evolution from zones to networks," Current Opin. Neurol, vol.25, 2012.

[5] Fredeik Edeluik, Bjorn Andersson, Stefan Jakobsson,stig Larsson, Mikael Persson, Yazdzn Shirvany,"An improved method for dipole modeling in EEG-based source localization",vol.15,2008.

[6] B. Vanrumste, G. V. Hoey, R. V. de Walle, M. R. D’Have, I. A. Lemahieu, and P. A. Boon, "Comparison of performance of spherical and realistic head models in dipole localization from noisy EEG," Med. Eng. Phys., vol. 24, no. 6, pp. 403-418, 2002.

[7] C. C. Wood, "Application of dipole localization methods to source identification of human evoked potentials," Ann. NY Acad. Sci., vol. 388 , no. 1 , pp. 139-155, 1982. 